\title{
Actualités proctologiques : conduite à tenir devant un prurit anal
}

\author{
Ph. GUYOT (Lyon)
}

Le prurit anal est l'ensemble des sensations cutanées péri-anales qui aboutissent au grattage. C'est un symptôme fréquent à prédominance masculine (sex ratio $=4$ ) et qui reste inconnue une fois sur deux.

C'est une région à risque car l'innervation sensitive est fortement développée et les facteurs locaux d'irritation sont nombreux : défécation, hygiène défaillante ou excessive, facteurs uro-gynécologiques associés, pathologie «de plis».

Sur le plan physio-pathologique, il existe deux grands types de cause : proctologique et dermatologique. Les pathologies susceptibles d'aboutir à un prurit anal sont des pathologies suintantes qui irritent la région cutanée marginale par le biais d'un écoulement purulent, muqueux ou fécaloïde, aboutissant à la sensation de prurit ou de brûlures qui entrainent un grattage, à l'origine de lésions cutanées qui facilitent la surinfection et l'hypersensibilisation génératrices de prurit. Secondairement, le risque est que le prurit évolue pour son propre compte alors que la cause elle-même a disparu.

\section{CLINIQUE}

1 - l'interrogatoire est minutieux :

- ancienneté, siège, extension, horaires et facteurs déclenchants;

- habitudes hygiéniques et vestimentaires;

- prises médicamenteuses;

- antécédents et terrain;

- signes fonctionnels associés ano-rectaux et généraux.

\section{2 - l'examen clinique est exhaustif :}

- inspecte en position genu pectoral ou décubitus latéral gauche;

- apprécie la réalité et l'intensité du prurit : excoriation, raghades des lésions récentes, lichénification des lésions anciennes;

- analyse les lésions marginales;

- doit être systématiquement associé à un examen proctologique et à un examen général complet.

\section{LES CAUSES DE PRURIT}

Dermatologique ou proctologique mais en différenciant causes et conséquences (dermite ou lésions ano-cutanées secondaires), causes et pathologies associées (hémorroïdes ou fissures chroniques «non pathologiques»). Il est nécessaire d'éliminer les causes générales avant d'envisager le prurit anal idiopathique qui est un diagnostic d'élimination.

Les causes dermatologiques comportent les dermites infectieuses bactériennes virales ou parasitaires ; les dermites spécifiques à forme anale ; les dermatoses irritatives.

Les causes proctologiques comportent toute la pathologie proctologique susceptible d'aboutir à des suintements : fistule bien sâr en priorité, mais également volumineuses hémorroïdes de stade IV ou troubles du fonctionnement recto-sphinctérien pouvant aboutir à des suintements fécaux : atteinte sphinctérienne, microrectite, troubles de la statique pelvienne et en particulier, prolapsus total du rectum, troubles du transit.

Les causes générales rares sont diabète, dysthyroïdie, insuffisance rénale, Hodgkin, polyglobulie, hyper-uricémie.

Certaines conditions vont favoriser la survenue d'un prurit : grossesse, ménopause.

A l'issue de cette recherche étiologique exhaustive, l'absence de cause aboutit une fois sur deux au diagnostic de prurit anal essentiel. Celui-ci est vraisemblablement essentiel par disparition de la cause et pérennisé par un auto-entretien. L'influence des facteurs psychiques sur l'apparition et la pérennisation du prurit n'a pas pu être démontré malgré de nombreux articles.

La conduite à tenir repose essentiellement sur des mesures d'hygiène locale, le but est d'obtenir une région propre et sèche sans excès de nettoyage mais avec un lavage soigneux de la région marginale après la selle en recourant éventuellement à des lingettes, par l'éviction de tous les facteurs allergisants locaux et l'utilisation prudente et rationnelle de crème topique : pâte à l'eau et crème hydratante, dermocorticoïde en utilisation brève et de classe IV en cas d'atteinte aiguë. En cas de dermite lichénifiée, corticoïdes de classe I relayés par une crème topique.

\section{RÉFÉRENCES}

1. BOTTERILL I.D., SAGAR M. - Intra-dermal methylene blue, hydrocortisone and lignocaine for chronic intractable pruritus ani. Colorectal Dis., 2002, 4, 144-146.

2. LYSY J., SISTIERY-ITTAH M., SHMUELI A., STRAUSSLIVIATAN N., MINDRUL V., KERET D. et al. - Topical capsaicin- a novel and effective treatment for idiopathic intractable pruritus ani : a randomised, placebo controlled, crossover study. Gut, 2003, 52, 1323-1326.

3. DANIEL G.L., LONGO W.E., VERNAVA A.M. $3^{\text {rd }}$ - Pruritus ani. Causes and concerns. Dis. Colon Rectum, 1994, 37, 670-674.
4. HANDA Y., WATANABE O., ADACHI A., YAMANAKA N. - Squamous cell carcinoma of the anal margin with pruritus ani of long duration. Dermatol. Surg., 2003, 29, 108-110.

5. YUHAN R., ORSAY C., DELPINO A., PEARL R., PULVIRENTI J., KAY S. et al. - Anorectal disease in HIV-infected patients. Dis. Colon Rectum, 1998, 41, 1367-1370.

6. SHUTZE W.P., GLEYSTEEN J.J. - Perianal Paget's disease. Classification and review of management : report of two cases. Dis. Colon Rectum, 1990, 33, 502-507. 